\title{
BMJ Open Impact of sex and myocardial function on association of obesity with mortality in Asian patients with acute heart failure: a retrospective analysis from the STRATS-AHF registry
}

\author{
Chan Soon Park, ${ }^{1,2}$ Jun-Bean Park, ${ }^{2,3}$ Jin Joo Park, ${ }^{2,4}$ Jae-Hyeong Park, ${ }^{5}$ \\ Goo-Yeong Cho (iD) 2,4
}

To cite: Park CS, Park J-B, Park JJ, et al. Impact of sex and myocardial function on association of obesity with mortality in Asian patients with acute heart failure: a retrospective analysis from the STRATS-AHF registry. BMJ Open 2020;10:e031608. doi:10.1136/ bmjopen-2019-031608

- Prepublication history and additional material for this paper are available online. To view these files, please visit the journal online (http://dx.doi. org/10.1136/bmjopen-2019031608).

CSP and J-BP contributed equally.

Received 12 May 2019 Revised 13 December 2019 Accepted 13 January 2020

Check for updates

(C) Author(s) (or their employer(s)) 2020. Re-use permitted under CC BY-NC. No commercial re-use. See rights and permissions. Published by BMJ.

For numbered affiliations see end of article.

Correspondence to

Dr Goo-Yeong Cho;

cardioch@snu.ac.kr

\section{ABSTRACT}

Objectives Impact of sex and myocardial function on the obesity paradox in heart failure (HF) is unknown. We explored whether sex, myocardial function, and left ventricular (LV) geometry explains the protective association of body mass index (BMI) with mortality, and investigated whether metabolic health status affects this association.

Design A multicentre cohort study with patients with acute HF admitted from January 2009 to December 2016 with a median follow-up of 33.7 months.

Setting Three tertiary hospitals.

Participants A total of 2021 overweight-to-obese (OW) and 1543 normal-weight (NW) patients with acute HF. Measurements The primary outcome was all-cause mortality. Patients were categorised as either OW $\left(\mathrm{BMl} \geq 23 \mathrm{~kg} / \mathrm{m}^{2}\right)$ or $\mathrm{NW}\left(\mathrm{BMl}<23 \mathrm{~kg} / \mathrm{m}^{2}\right)$. BMl was used as both categorical and continuous variables. Clinical, laboratory and echocardiographic measures, including LV global longitudinal strain (LV-GLS), LV-ejection fraction, LV geometry, were obtained.

Results During the follow-up period, 1392 patients died (685 OW and $707 \mathrm{NW}$ ). BMI was significantly associated with mortality in univariate $\left(\mathrm{HR}=0.929\right.$ per $\mathrm{kg} / \mathrm{m}^{2}$, $\mathrm{p}<0.001)$ and multivariate analyses $\left(\mathrm{HR}=0.954 \mathrm{per} \mathrm{kg} / \mathrm{m}^{2}\right.$, $\mathrm{p}<0.001)$. In multivariable fractional polynomials, higher BMls were associated with lower mortality overall and in subgroups by sex, LV-GLS and LV geometry, with a steeper association in men ( $p$-interaction $<0.001$ ). In women, there were significant interactions of BMI with LV-GLS $(p$-interaction $=0.044)$ and age $(p$-interaction $=0.040)$ for mortality; the protective association of BMI with mortality was confined to subgroups with high LV-GLS (>10.1\%) or elderly patients ( $\geq 75$ years). In men, this association was found in all subgroups without significant interaction. Metabolically healthy obese patients had better survival than metabolically unhealthy obese patients (log-rank $p<0.001)$. Conclusions In women, a significant interaction was observed between BMI and age or LV-GLS in association with mortality, suggesting that sex, ageing and myocardial dysfunction can affect the magnitude of the obesity paradox in HF. Metabolic health status provides prognostic information beyond obesity status.

\section{Strengths and limitations of this study}

- We analysed comprehensive echocardiographic parameters including left ventricular (LV) global longitudinal strain, LV ejection fraction and LV geometry according to sex to investigate the obesity paradox in heart failure (HF).

- This study collected a variety of demographic data and medical history and performed sensitivity analyses to reduce possible biases.

- Echocardiographic images were transferred to the strain core laboratory, and strain measurements were conducted by independent observers blinded to participants' clinical information and the study design.

- We used body mass index as a parameter of obesity for analyses, but did not assess body fat mass or cardiopulmonary fitness.

- This is a cohort study, although a large one, in which unmeasured confounding is an inherent limitation.

Trial registration number Registry: ClinicalTrials.gov Number: NCT03513653 (https://clinicaltrials.gov/ct2/show/ NCT03513653)

\section{INTRODUCTION}

There is a consensus that efforts to prevent and treat obesity are needed to reduce the heart failure (HF) burden for patients and society. ${ }^{1}$ Regarding the association of obesity with prognosis in patients with HF, however, substantial data have demonstrated counterintuitive finds, the so-called 'obesity paradox', where overweight-to-obese (OW) patients with $\mathrm{HF}$ have more favourable outcomes. ${ }^{2} 3$ Increasing attention has been paid to whether the obesity paradox exists in the overall HF population, and whether this phenomenon is confined to specific subgroups, ${ }^{45}$ since the answers to these questions have important 
implications for the value of intentional weight loss to improve prognosis.

The effect of left ventricular (LV) systolic function on the obesity paradox has been widely explored, as it is an important predictor of survival in $\mathrm{HF}$, but with contradicting results. ${ }^{46}$ These controversial findings might be explained by the method used to assess LV systolic function in previous studies (LV ejection fraction, LV-EF). ${ }^{467}$ Considering that alterations in $\mathrm{LV}$ wall thickness and/or volume compensate for impaired LV shortening, LV-EF, a volume-based parameter, cannot accurately reflect $\mathrm{LV}$ systolic function in patients with abnormal LV geometry. ${ }^{7}$ Since obesity is frequently associated with adverse LV remodelling, ${ }^{3} 89$ the prognostic value of LV-EF in obese patients could be confounded by LV geometrical patterns. Given that LV strain, an index of myocardial deformation, reflects LV systolic function better than LV-EF in the presence of geometrical confounders, ${ }^{7}$ it may provide superior prognostic information to LV-EF in obese patients.

Hence, we hypothesised that the obesity paradox in HF would be present in patients with preserved LV systolic function, but not in those with impaired LV systolic function, as stratified by the values of the LV strain. We also postulated that, as suggested previously, ${ }^{510}$ there might be a sex-related difference in the obesity paradox of $\mathrm{HF}$, especially LV strain is considered together with sex. Additionally, we investigated the effect of metabolically healthy obesity on prognosis in patients with HF in the hope of paving the way for future therapeutic implications of managing metabolic abnormalities.

\section{METHODS}

\section{Study design and participants}

The STrain for Risk Assessment and Therapeutic Strategies in patients with Acute Heart Failure registry protocol has been previously described. ${ }^{11}$ Briefly, from January 2009 through December 2016, we recruited 4312 consecutive patients hospitalised for acute HF from three tertiary university hospitals. Inclusion criteria were patients who had compatible symptoms and signs of HF and at least one of the following: (1) Pulmonary oedema defined as rales on physical examination or congestion on chest radiography. (2) Objective findings of LV systolic dysfunction or structural heart disease. The lack of data on LV global longitudinal strain (GLS) was a main exclusion criterion; echocardiography was performed in $4237(98.3 \%)$ patients, and LV-GLS was measured in 4088 subjects $(96.4 \%)$. Patients without body mass index (BMI) data $(n=130)$ were also excluded. We further excluded underweight participants (BMI $<18.5 \mathrm{~kg}$ / $\left.\mathrm{m}^{2}\right)(\mathrm{n}=394)$, based on previous studies suggesting that underweight subjects might confound the association between obesity and mortality. ${ }^{12} 13$ The final sample was 3564 patients. This study complied with the Declaration of Helsinki. The need for written informed consent was waived.

\section{Data collection}

BMI was calculated as body weight divided by height squared $\left(\mathrm{kg} / \mathrm{m}^{2}\right)$ on admission. The WHO classification for Asian populations was used to categorise patients according to their obesity status; ${ }^{14}$ the normal-weight (NW) group was defined as patients with BMI of 18.5$22.9 \mathrm{~kg} / \mathrm{m}^{2}$ and OW group as those with BMI $\geq 23 \mathrm{~kg} / \mathrm{m}^{2}$ (overweight as BMI $23.0-24.9 \mathrm{~kg} / \mathrm{m}^{2}$ and obese as BMI $\left.\geq 25.0 \mathrm{~kg} / \mathrm{m}^{2}\right)$. Hypertension was defined by the presence of elevated systolic $(\geq 140 \mathrm{~mm} \mathrm{Hg})$ and/or diastolic $(\geq 90$ $\mathrm{mm} \mathrm{Hg}$ ) blood pressure, a history of hypertension or the use of antihypertensive agents. Diabetes mellitus was defined by the presence of elevated fasting glucose level $(\geq 126 \mathrm{mg} / \mathrm{dL})$, a history of diabetes mellitus or the use of antidiabetic agents. Dyslipidaemia was defined by the presence of elevated serum total cholesterol ( $\geq 200 \mathrm{mg}$ / $\mathrm{dL}$ ), a history of dyslipidaemia or the use of cholesterollowering medications. In this study, the metabolically healthy subgroup was defined as patients without any hypertension, diabetes mellitus and dyslipidaemia.

The primary outcome was all-cause mortality. Mortality data were obtained and verified via the centralised database of national death records.

\section{Echocardiographic and strain analysis}

Echocardiographic examinations were performed according to an established guideline. ${ }^{15} \mathrm{LV}$ geometry was classified as normal, concentric remodelling, concentric hypertrophy or eccentric hypertrophy as previously described. ${ }^{16}$ Echocardiographic images were transferred to the strain core laboratory for strain analysis, and strain analysis was performed as previously described. ${ }^{11}$ As LV-GLS is a negative value, the absolute value $|x|$ was used for simpler interpretation. All strain measurements were conducted by independent observers blinded to participants' clinical information and the study design. The median LV-GLS (10.1\%) was used to stratify patients.

\section{Statistical analysis}

For descriptive statistics, data were presented as numbers and relative frequencies for categorical variables and as mean $\pm \mathrm{SD}$ for continuous variables. Data of B-type natriuretic peptide (BNP) and N-terminal proBNP (NT-proBNP) were presented as median with IQR due to their skewed distribution. For the comparison between groups, the $\chi^{2}$ test or Fisher's exact test was used for categorical variables as appropriate, and the unpaired Student's $t$-test was applied for continuous variables. For survival analyses, we performed Kaplan-Meier and Cox proportional hazards regression analyses. The chronological trend of all-cause mortality was expressed as KaplanMeier estimates with log-rank test to compare survival between groups. Cox proportional hazards regression models were used to determine effects of BMI on allcause mortality while adjusting for potential confounders. Testing of validity of proportional hazards assumption was performed using Schoenfeld residuals. We included variables associated with mortality with a value of $p<0.05$ 
Table 1 Baseline characteristics

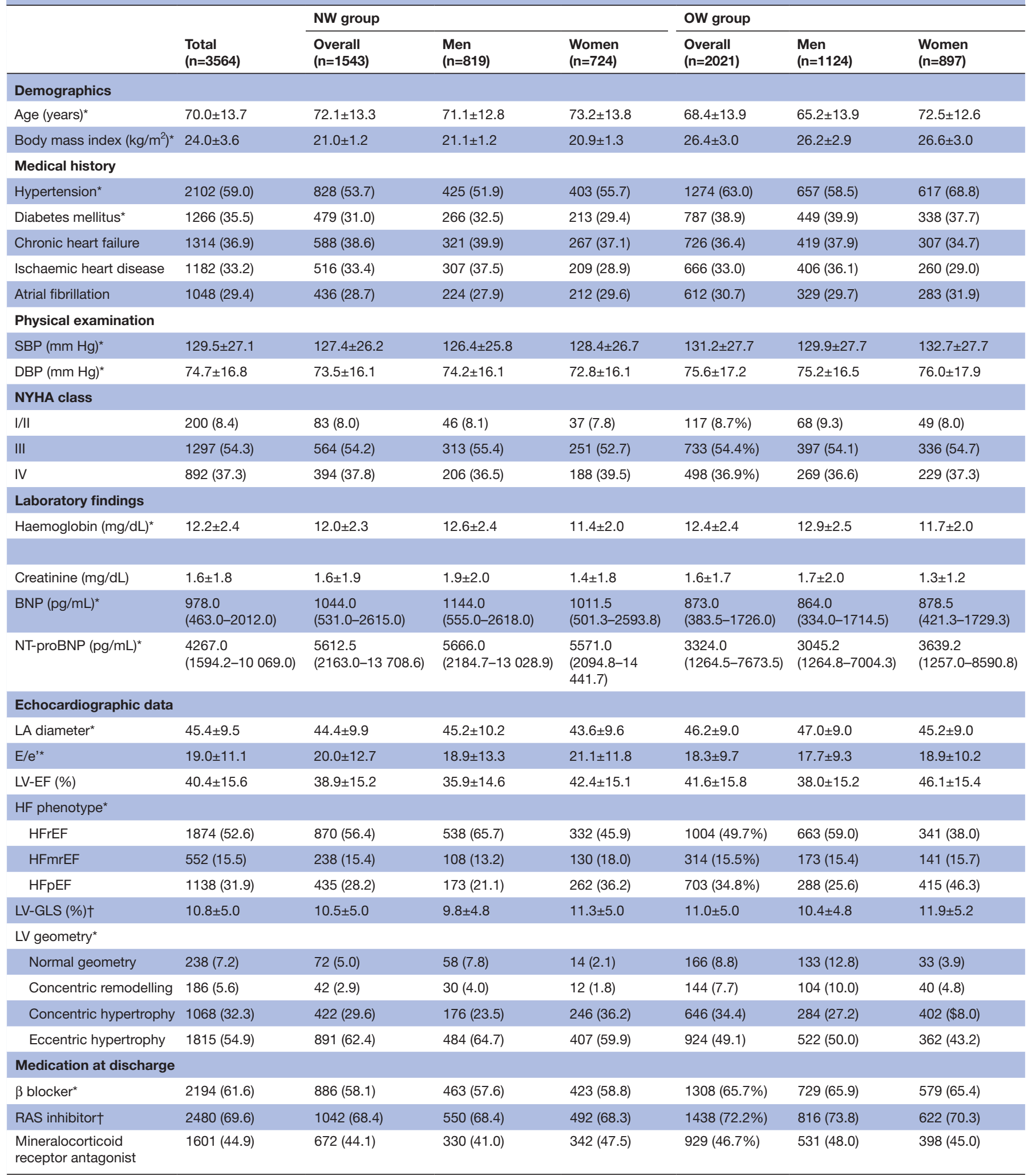

Values given as number (percentage), mean ${ }_{ \pm} \mathrm{SD}$ or median $(\mathrm{IQR})$ unless otherwise indicated.

$\mathrm{HF}$ phenotypes are defined as follows: HFrEF if the LV-EF is $<40 \%$, HFmrEF if the LV-EF is between $40 \%$ and $49 \%$, and $\mathrm{HFpEF}$ if the LV-EF is $\geq 50 \%$.

${ }^{*} \mathrm{p}<0.001$ (between NW and OW groups).

tp $<0.01$ (between NW and OW groups).

DBP, diastolic blood pressure; EF, ejection fraction; GLS, global longitudinal strain; HF, heart failure; HFmrEF, heart failure with mid-range ejection fraction; HFpEF, heart failure with preserved ejection fraction; HFrEF, heart failure with reduced ejection fraction; LA, left atrial; LV, left ventricular; NW, normal weight; NYHA, New York Heart Association; OW, overweight-to-obese; RAS, renin-angiotensin system inhibitor; SBP, systolic blood pressure. 
in univariate analysis and as covariates in multivariate analysis. The association between BMI and mortality was assessed by the Cox regression model, and non-linearities were explored using multivariable fractional polynomials. ${ }^{16}$ For all-cause mortality, sex-specific subgroup and interaction-term analyses were performed to explore potential effect modifiers. In sensitivity analyses, a propensity-score-matched population was selected using the nearest neighbour method without replacement to adjust for uneven distribution of baseline characteristics.

Two-sided values of $p<0.05$ were considered statistically significant. Statistical tests were performed using STATA software (V.12, Stata Corp).

\section{Patient and public involvement}

The participants were not involved in the development of the research question, outcome measures, design, recruitment and conduct of this study.

\section{RESULTS}

\section{Baseline characteristics}

Table 1 summarises baseline characteristics of 3564 study subjects by obesity status and sex. The proportions in the NW and OW groups were $43.3 \%$ and $56.7 \%$, respectively. Among the OW group, $39.6 \%$ were overweight and $60.4 \%$ were obese. The values of LV-GLS and LV-EF and the proportion of concentric LV remodelling/hypertrophy were significantly higher in OW than NW patients. When stratified by both obesity status and sex, OW women had significantly higher LV-GLS values than their male counterparts $(\mathrm{p}<0.001)$.

We matched 2978 patients on the propensity score, and their baseline characteristics were balanced without any significant difference (online supplementary table 1).

\section{Distribution of LV-GLS and death rates according to obesity status and LV geometry}

When we assessed the proportion of each LV geometrical pattern by obesity status, normal geometry and concentric remodelling/hypertrophy was more prevalent in OW $(8.8 \%$ and $42.0 \%)$ than in NW patients $(5.0 \%$ and $32.5 \%)$, but eccentric hypertrophy was less frequently observed in OW $(49.1 \%)$ than NW patients $(62.4 \%)$ (online supplementary figure 1A). Compared with patients with normal LV geometry (NW group: LV-GLS 12.5; OW group: 12.6), the mean LV-GLS was similar in patients with concentric remodelling/hypertrophy (NW group: LV-GLS 12.2, $\mathrm{p}=0.675$; OW group: $12.1, \mathrm{p}=0.217$ ) and significantly lower in those with eccentric hypertrophy (NW group: LV-GLS 9.6; OW group: 10.1, $\mathrm{p}<0.001$, all), regardless of obesity status (online supplementary figure 1B). The LV-GLS value measured in the eccentric hypertrophy subgroup was significantly higher in OW patients than in NW patients ( 10.1 vs $9.6, \mathrm{p}=0.030$ ), whereas that measured in the normal and concentric remodelling/hypertrophy subgroups did not significantly differ between the groups. The mortality rate increased gradually, being lowest in patients with normal geometry, higher in patients with concentric remodelling/hypertrophy and highest in patients with eccentric hypertrophy. It was lower in OW $(21.1 \%, 32.2 \%$ and $37.2 \%)$ than NW patients $(29.2 \%$, $43.6 \%$ and $46.6 \%$ ) across all LV geometrical patterns (online supplementary figure 1C).

\section{Association between BMI and mortality}

The mortality risk decreased as BMI increased in study subjects, which was in accordance with the obesity paradox (online supplementary figure 2A). A similar association was observed in subgroups classified by the LV geometrical pattern (online supplementary figure 2B,D). The association of higher BMI with lower mortality was found regardless of sex, although the slope of this relationship was significantly flatter in women than in men (p-interaction $<0.001$ ) (online supplementary figure $2 \mathrm{E}$ ). In subgroup analyses stratified by LV-GLS, there was no significant interaction between LV-GLS and BMI for mortality (p-for interaction $=0.493)$ (online supplementary figure $2 \mathrm{~F}$ ).

\section{Mortality for overall patients and subgroups}

Among overall patients, 1392 (39.1\%) died during a median follow-up of 33.7 months (IQR, 13.7-55.5). The LV-GLS value was significantly lower in deceased patients than survivors $(9.9 \pm 4.8$ vs $11.4 \pm 5.0, \mathrm{p}<0.001)$ but LV-EF was not $(40.8 \pm 15.6$ vs $39.9 \pm 15.5, \mathrm{p}=0.100)$ (online supplementary table 2$)$.

In the NW group, women had significantly higher survival rates than men $(\log$-rank $\mathrm{p}=0.013)$, while in the OW group, there was no significant sex-related difference in survival (log-rank $\mathrm{p}=0.446$ ) (figure 1A). When we classified patients into two subgroups by the median LV-GLS, patients with low LV-GLS had significantly higher mortality in the NW $(\log$-rank $\mathrm{p}<0.001)$ and OW groups (log-rank $\mathrm{p}<0.001$ ) (figure 1B). LV-EF did not stratify the mortality risk in either group (online supplementary figure $3 A, B$ ). When stratified by LV geometry and obesity status, survival was highest in patients with normal geometry in the NW and OW groups (Figure 1C and online supplementary figure $3 \mathrm{C}, \mathrm{D}$ ). The survival rate was lowest in patients with eccentric hypertrophy, but its difference with concentric remodelling/hypertrophy became less marked over time and similar after a follow-up of 60 months in the NW group (online supplementary figure 3C) and 80 months in the OW group (online supplementary figure 3D). The mortality risk was significantly lower in patients with metabolically healthy obesity than those with metabolically unhealthy obesity (online supplementary figure $3 \mathrm{~F}$ ), and a smaller but significant increase in mortality risk was noted in NW patients with metabolic abnormalities compared with those without (online supplementary figure 3E). Similar results were found in sensitivity analyses with the propensity-score-matched population (online supplementary figure 4).

Table 2 presents the results from the Cox models. BMI was significantly associated with mortality in both univariate analysis $(0.929,95 \%$ CI 0.914 to $0.944, \mathrm{p}<0.001)$ 
(A)

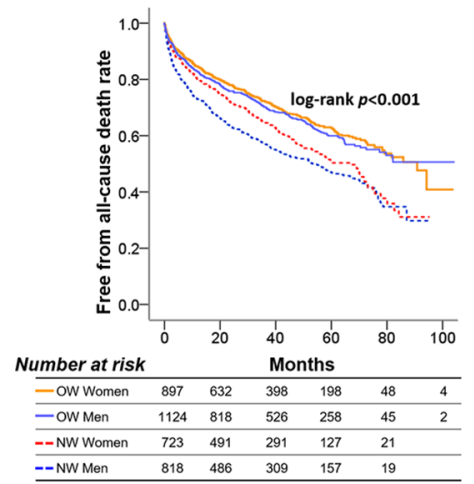

(B)

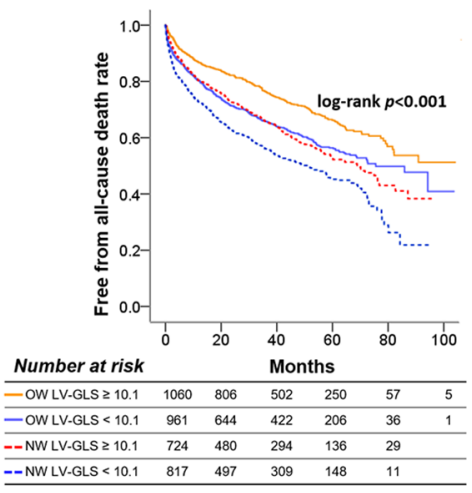

(C)

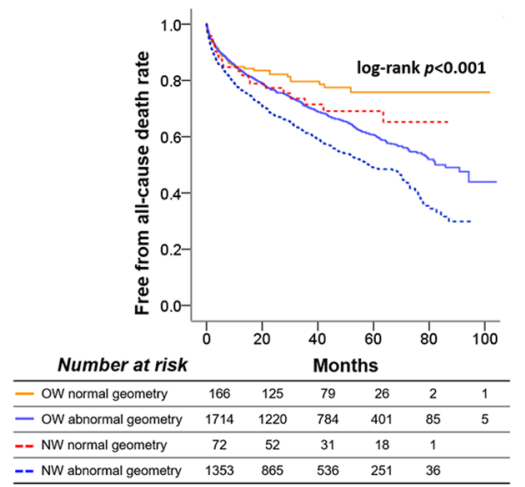

Figure 1 Kaplan-Meier curves demonstrating the mortality risk by sex (A), LV-GLS (B) or LV geometry (C) in two subgroups stratified by obesity status. GLS, global longitudinal strain; LV, left ventricle; NW, normal weight; OW, overweight-to-obese.

and multivariate analysis $(0.954,95 \%$ CI 0.937 to 0.971 , $\mathrm{p}<0.001)$. In multivariate analyses, LV-GLS was a significant independent determinant for mortality (HR $0.943,95 \%$ CI 0.932 to $0.954, p<0.001)$. Conversely, LV-EF did not remain a significant predictor for mortality in multivariate analysis, although significant in univariate analysis (HR 0.995, 95\% CI 0.992 to $0.999, \mathrm{p}=0.006$ ). Age, sex and BMI were independent significant predictors of mortality. Similar results were found when analyses were performed separately in men and women (online supplementary table 3). In both men and women, BMI was a significant predictors of mortality in univariate analyses (HR $0.902,95 \%$ CI 0.881 to $0.924, \mathrm{p}<0.001$ for male; HR $0.956,95 \%$ CI 0.935 to 0.979 , $\mathrm{p}<0.001$ for female) as well as multivariate analyses (HR $0.939,95 \%$ CI 0.914 to $0.963, \mathrm{p}<0.001$ for male; HR 0.967 , $95 \%$ CI 0.945 to $0.990, \mathrm{p}<0.001$ for female).

\section{Risk stratification}

In multivariate analyses, there was an independent association of sex and BMI with mortality (figure 2A). The highest mortality was noted among NW men, followed by NW women and OW men, while the lowest mortality was found in $\mathrm{OW}$ women. When we stratified patients into four subgroups according to LV-GLS and BMI, there was a graded, independent association between the mortality risk and the presence of lower BMI and/ or LV-GLS (figure 2B). OW patients with high LV-GLS were at the lowest mortality risk (reference), and NW patients with high LV-GLS, OW patients with low LV-GLS and NW patients with low LV-GLS were at progressively higher risk $(p$ trend $<0.001)$. The difference between OW patients with low LV-GLS and NW patients with high LV-GLS was not significant. When stratified by LV geometrical patterns, the mortality risk was highest among NW patients with abnormal LV geometry compared with the other groups (figure 2C). Metabolically unhealthy NW patients had the highest mortality, followed by metabolically unhealthy OW patients, metabolically healthy NW patients and metabolically healthy OW patients (online supplementary figure 5).

\section{Sex-specific subgroup analyses}

Figure 3 shows the association of BMI, per $1 \mathrm{~kg} / \mathrm{m}^{2}$ increment, with mortality in men and women. In men, a greater BMI was related to favourable survival across all subgroups within HF (figure 3A). However, in women, significant heterogeneity was found when examined as a function of LV-GLS or age (figure 3B); the protective association of BMI with mortality was confined to women with high LV-GLS or elderly patients.

Table 2 Cox-proportional hazards regression analysis for all-cause mortality

\begin{tabular}{|c|c|c|c|c|c|c|}
\hline & \multicolumn{3}{|c|}{ Unadjusted } & \multicolumn{3}{|c|}{ Adjusted* } \\
\hline & HR & $95 \% \mathrm{Cl}$ & $P$ value & HR & $95 \% \mathrm{Cl}$ & P value \\
\hline \multicolumn{7}{|l|}{ All patients $(n=3564)$} \\
\hline \multicolumn{7}{|l|}{ Men $(n=1943)$} \\
\hline Body mass index $\left(\mathrm{kg} / \mathrm{m}^{2}\right)$ & 0.902 & 0.881 to 0.924 & $<0.001$ & 0.939 & 0.914 to 0.963 & $<0.001$ \\
\hline \multicolumn{7}{|l|}{ Women $(n=1621)$} \\
\hline Body mass index $\left(\mathrm{kg} / \mathrm{m}^{2}\right)$ & 0.956 & 0.935 to 0.979 & $<0.001$ & 0.967 & 0.945 to 0.990 & 0.006 \\
\hline
\end{tabular}

*Multivariate-adjusted HR was calculated after adjustment for age, sex, body mass index, diastolic blood pressure, diabetes mellitus, ischaemic heart disease, LV ejection fraction, LV global longitudinal strain, $\beta$-blocker, RAS inhibitor and MR antagonist.

$\mathrm{LV}$, left ventricular; MR, mineralocorticoid receptor; RAS, renin-angiotensin system inhibitor. 
(A)

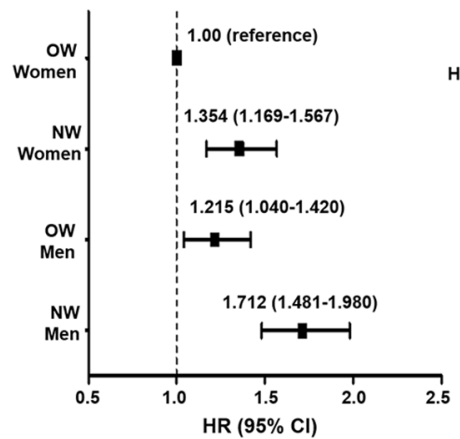

(B)

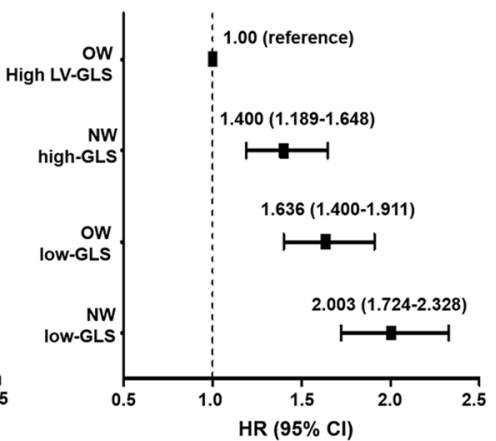

(C)

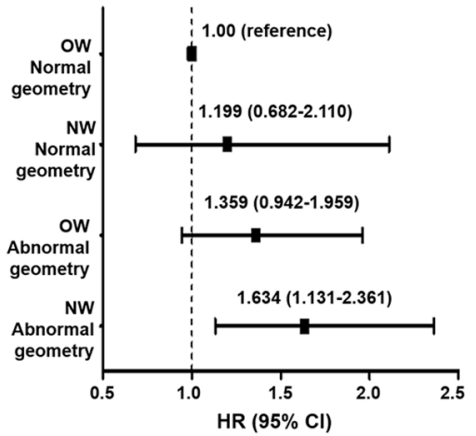

Figure 2 Forest plots of adjusted HRs depicting mortality in four subgroups categorised by BMI and sex (A), LV-GLS (B) or LV geometry (C). HRs were adjusted for the independent variables shown in table 2. BMI, body mass index; GLS, global longitudinal strain; LV, left ventricle; NW, normal weight; OW, overweight-to-obese.

(A)

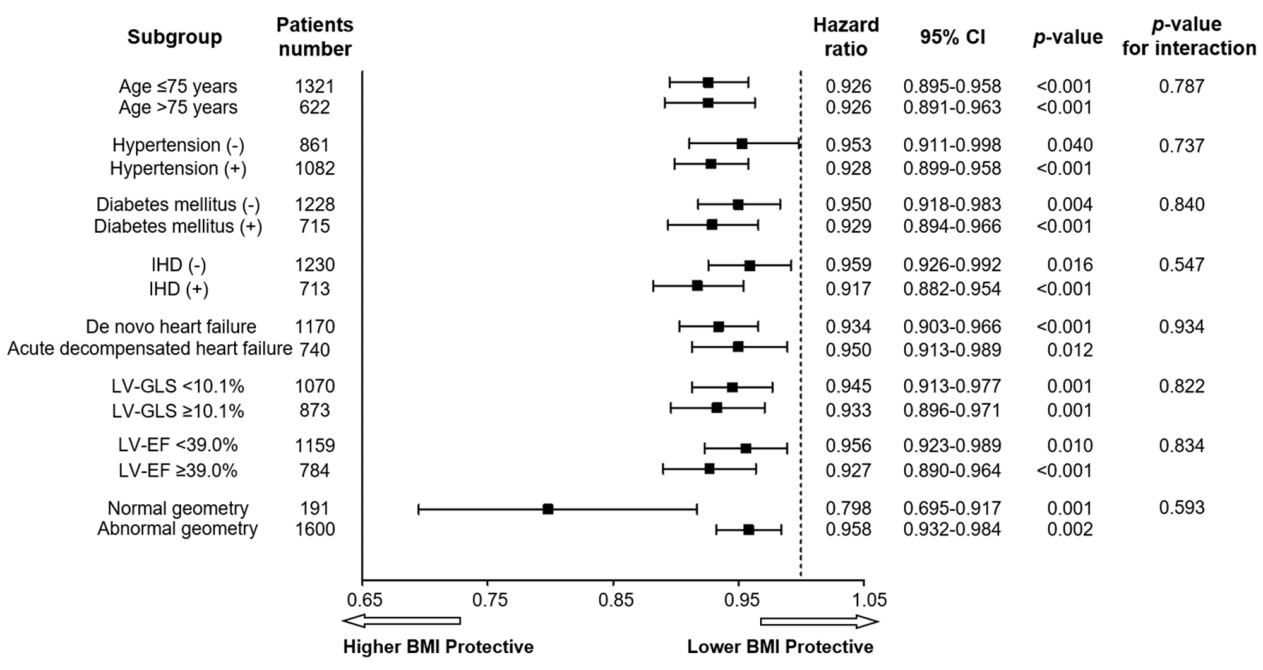

(B)

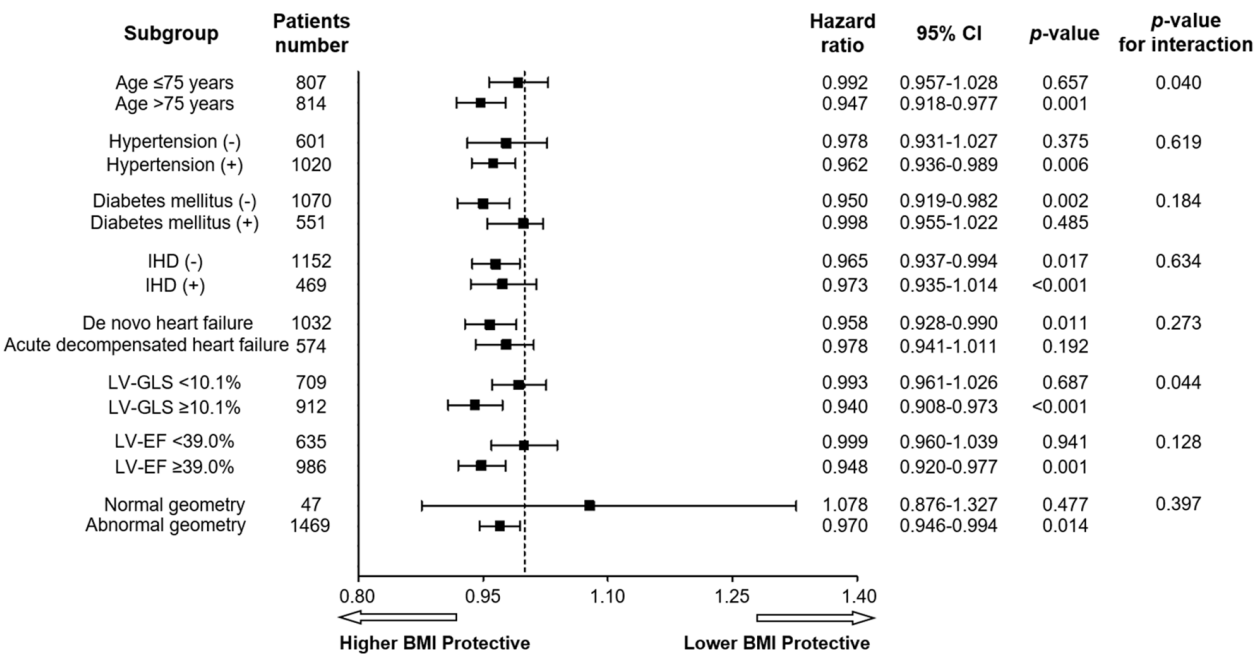

Figure 3 Forest plots of adjusted HRs for the relationship of BMI, per $1 \mathrm{~kg} / \mathrm{m}^{2}$ increment as a continuous variable, with allcause mortality in men (A) and women (B). The HR within each stratum was adjusted for the independent variables shown in table 2. BMI, body mass index; EF, ejection fraction; GLS, global longitudinal strain; LV, left ventricle 


\section{DISCUSSION}

The main findings of our study were as follows: (1) Compared with NW patients, OW patients had a smaller proportion of eccentric LV hypertrophy and better myocardial function and a lower morality rate. (2) In the overall population, the obesity paradox in HF was present irrespective of LV-GLS and LV geometry, with a steeper association among patients with high LV-GLS than those with low LV-GLS. (3) In women, a significant interaction was found between BMI and age or LV-GLS for mortality. (4) There was a stepwise, independent association between mortality and the presence of lower BMI and/or LV-GLS. (5) Metabolically healthy obese patients had better survival than metabolically unhealthy obese patients.

Importance of accurate assessment of LV systolic function for research on the obesity paradox in HF

Although obesity adversely affects LV structure and function in subjects without overt cardiovascular diseases (CVDs), ${ }^{17}$ it is paradoxically associated with improved prognosis in patients with HF. ${ }^{818}$ Several studies have suggested that the obesity paradox seems confined to certain subgroups of HF. ${ }^{16}$ This concept has important clinical implications because it can be translated into more individualised treatment strategies for HF by identifying specific subgroups of patients with HF who benefit from different therapeutic approaches, such as weight reduction versus nutritional interventions. However, a previous study showed that the obesity paradox was not present in patients with preserved LV-EF, ${ }^{4}$ while another study demonstrated that it was observed in those with preserved and reduced LV-EF. ${ }^{6}$ These contradicting results may stem from the limitation of LV-EF to assess LV systolic function in obese populations. The value of LV-EF as a measure of LV systolic function has been questioned in patients with concentrically remodelled LV. ${ }^{7}$ Further, obese patients had a distinct phenotype of $\mathrm{HF}$ with preserved $\mathrm{EF}$, including greater concentric LV remodelling. ${ }^{19}$ The prognostic value of LV-EF can be reduced when the study population includes a sizeable proportion of obese patients. Although the LV-GLS value in OW patients with eccentric hypertrophy was lowest among the four groups of OW patients, it was significantly higher than NW patients with eccentric hypertrophy. Compared with NW patients with HF, OW patients had a significantly lower proportion of eccentric hypertrophy, for which the mortality rate is very high. These findings raise the possibility that the much lower overall mortality observed in OW patients with HF may be partially due to more preservation of myocardial function and less prevalence of eccentric hypertrophy in this population. Important differences and new findings of our study in comparison with previous ones are summarised in table 3 .

\section{Impact of LV geometry on the obesity paradox in HF}

Although LV-GLS values were similar between patients with normal LV geometry and those with concentric remodelling/hypertrophy, the mortality rate was lowest in this group, implying that LV geometry should also

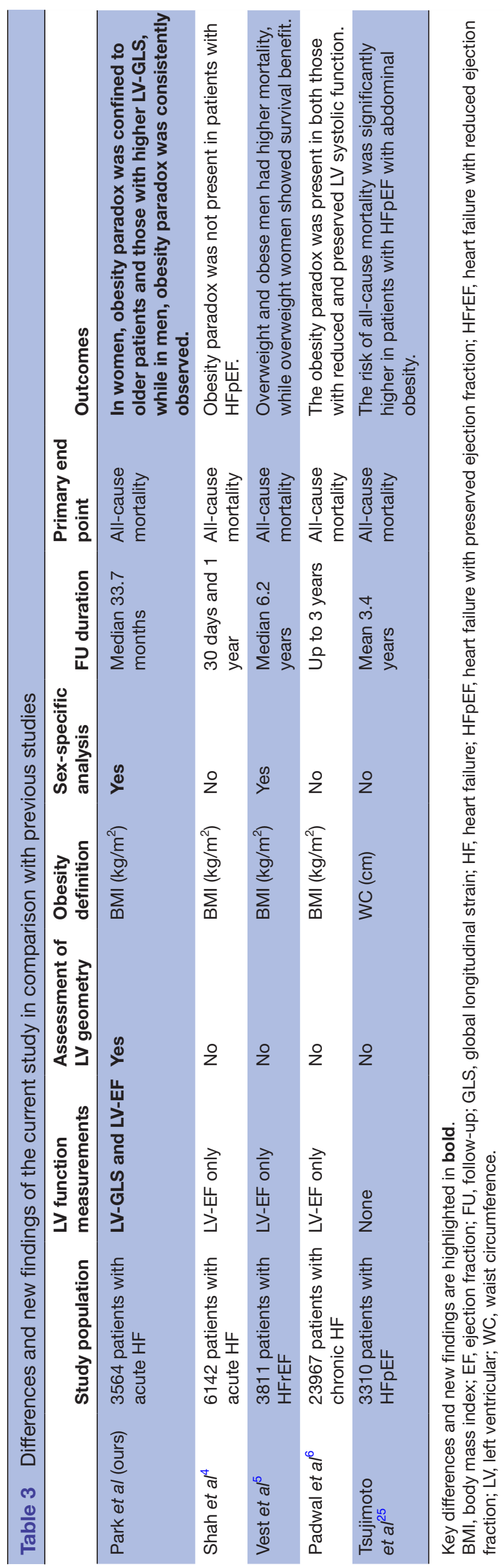


be considered when assessing the effect of LV-GLS on prognosis of patients with HF. We speculated that the development of concentric remodelling/hypertrophy accompanied by the preservation of LV-GLS offers a compensatory mechanism in $\mathrm{HF}$, being associated with a relatively lower mortality rate, but as HF further progresses, the myocardium enters the decompensated stage and eccentric hypertrophy with the substantial decrease in LV-GLS occurs, resulting in a higher mortality risk. Therefore, obesity can play an important role in delaying the transition from compensatory concentric remodelling/hypertrophy with preserved LV-GLS to decompensated eccentric hypertrophy with reduced LV-GLS. This speculation may be supported by previous research reporting that adipose tissue can provide protective benefits by producing soluble tumour necrosis factoralpha $(\mathrm{TNF}-\alpha)$ receptors that neutralise the detrimental effects of TNF- $\alpha$ on myocardial function. ${ }^{18}$

\section{Utility of LV-GLS for improved risk stratification in HF}

One important, novel finding of our study was that LV-GLS can improve risk stratification in this population beyond the obesity status. OW patients with HF generally had a better prognosis than NW ones, and there was no significant difference in mortality between OW patients with low LV-GLS and NW patients with high LV-GLS. In addition, patients with high LV-GLS had a steeper association compared with those with low LV-GLS, though the difference was not statistically significant between these groups. These findings suggest that more accurate identification of patients with impaired LV systolic function, by using LV-GLS assessment, may help stratify HF populations.

\section{Possible mechanisms for a sex-related difference in the obesity paradox in HF}

Previous studies have suggested that the obesity paradox in HF can differ by sex..$^{510}$ The obesity paradox in HF seems more prominent in men than in women, ${ }^{10}{ }^{20}$ which supports our findings. Conversely, Vest et a $\tilde{e}$ reported that the significant association of higher BMI with a survival benefit was found only in women. Our study suggests a possible explanation for this; the difference in the proportion of women with myocardial dysfunction included in analyses may affect the study results. According to our findings, the obesity paradox would be more evident in women when a greater proportion of women with higher LV-GLS values is included in the analysis. Although highly speculative, one might anticipate that the effect of efforts to modify BMI may differ by sex and myocardial function and therefore its benefit can be maximised by identifying specific patient subgroups for whom purposeful weight loss will provide the most benefit.

\section{Prognostic value of metabolic health status and its clinical implication}

Obesity increases CVD risk regardless of metabolic status. ${ }^{21}$ It has also been suggested that metabolically healthy obesity increases the risk of developing $\mathrm{HF}$ in individuals free of CVDs. ${ }^{22}$ However, the effects of metabolically healthy obesity on prognosis in subjects with CVDs, including HF, remain uncertain. We demonstrated that the mortality risk was significantly lower in patients with metabolically healthy obesity than those with metabolically unhealthy obesity, suggesting that metabolically healthy obesity may have a more protective effect on survival in patients with HF, and therefore, efforts to manage metabolic abnormalities in OW patients with HF may improve prognosis.

\section{Study limitations}

First, we used BMI as a parameter of obesity for analyses, which cannot differentiate body fat from muscle mass. Previous studies showed BMI was highly correlated with alternative measures of fat mass, ${ }^{23}$ and the obesity paradox in $\mathrm{HF}$ was present with BMI and body fat. ${ }^{24}$ In addition, central obesity, which could be evaluated by waist circumference or waist-to-hip ratio, has been reported to be a better predictor of CVDs, such as myocardial infarction and stroke, than BMI. The assessment of central obesity can provide additional prognostic information beyond BMI in $\mathrm{HF}$, as in other CVDs. However, our study did not assess these measures of central obesity, and therefore further studies are warranted to determine the association between central obesity, sex and myocardial function regarding mortality in patients with HF, which will provide additional insight into the obesity paradox in $\mathrm{HF}^{25}$ Second, we could not assess cardiopulmonary fitness and physical activity. Cardiopulmonary fitness has been reported to be associated with clinical outcomes. ${ }^{26}{ }^{27}$ Physical activity, a prognostic factor in patients with coronary heart disease, ${ }^{28}{ }^{29}$ might have similar prognostic value in patients with HF. Considering all together, ${ }^{30}{ }^{31}$ further studies are needed to assess the effects of sex, myocardial function, fitness and physical activity on the obesity paradox in HF. In addition, we used body weight and height assessed by nurses on admission for our analyses, which might partially reflect volume status, and thus confound our results. Considering that increased volume overload might result in both increased body weight and increased mortality, attenuating the protective association of BMI with mortality, the use of body weight measured on admission would not materially change the major conclusions of the study. As we have enrolled and analysed patients from East Asia, it is uncertain whether our findings could be generalised to other ethnicities. In addition, as we comprehensively investigated the association between BMI and various variables for mortality, there might be a possibility of type 1 errors owing to the large number of possible interaction tests performed. Lastly, we excluded underweight patients to minimise the confounding effects of cachexia or unintentional weight loss due to advanced HF status or other comorbidities on study outcomes. Further studies are needed to explore the association between sex, myocardial function, body mass index and mortality in these patients.

\section{CONCLUSIONS}

In the overall population, the obesity paradox in HF was present irrespective of LV-GLS and LV geometry. In women, 
the protective association of higher BMI on prognosis of HF was confined to older patients and those with higher LV-GLS, while in men, the obesity paradox was consistently observed across all subgroups. These findings could suggest that sex, age and the degree of myocardial dysfunction can have an influence on the magnitude of the obesity paradox in HF, and thus sex-specific risk stratification of patients with HF, accompanied by accurate assessment of myocardial function, is needed to improve the prognosis. Patients with metabolically healthy obesity had better survival than those with metabolically unhealthy obesity, suggesting prognostic and therapeutic implications of metabolic abnormalities in OW patients with HF.

\section{Author affiliations}

${ }^{1}$ Graduate school of Medical Science and Engineering, Korea Advanced Institute of Science and Technology, Daejeon, Republic of Korea

${ }^{2}$ Department of Internal Medicine, Seoul National University College of Medicine, Seoul, Republic of Korea

${ }^{3}$ Cardiovascular Center, Seoul National University Hospital, Seoul, Republic of Korea ${ }^{4}$ Cardiovascular Center, Department of Internal Medicine, Seoul National University Bundang Hospital, Seongnam, Republic of Korea

${ }^{5}$ Department of Cardiology in Internal Medicine, Chungnam National University Hospital, Chungnam National University College of Medicine, Daejeon, Republic of Korea

Contributors CSP: Conceptualisation, statistical analysis and manuscript writing. J-BP: Conceptualisation, recruiting patients, collecting samples, statistical analysis and manuscript writing. JJP: recruiting patients, collecting samples and manuscript review. J-HP: recruiting patients, collecting samples and manuscript review. G-YC: Conceptualisation, recruiting patients, collecting samples, statistical analysis and manuscript review. All authors read and approved the manuscript.

Funding The authors have not declared a specific grant for this research from any funding agency in the public, commercial or not-for-profit sectors.

Competing interests None declared.

Patient consent for publication Not required.

Ethics approval The study protocol was approved by the ethics committee at each institute, that is, Seoul National University Hospital, Seoul National University Bundang Hospital and Chungnam National University Hospital.

Provenance and peer review Not commissioned; externally peer reviewed.

Data availability statement Data are available upon reasonable request.

Open access This is an open access article distributed in accordance with the Creative Commons Attribution Non Commercial (CC BY-NC 4.0) license, which permits others to distribute, remix, adapt, build upon this work non-commercially, and license their derivative works on different terms, provided the original work is properly cited, appropriate credit is given, any changes made indicated, and the use is non-commercial. See: http://creativecommons.org/licenses/by-nc/4.0/.

ORCID iD

Goo-Yeong Cho http://orcid.org/0000-0002-7067-5535

\section{REFERENCES}

1 Kenchaiah S, Evans JC, Levy D, et al. Obesity and the risk of heart failure. N Engl J Med 2002;347:305-13.

2 Lavie CJ, Sharma A, Alpert MA, et al. Update on obesity and obesity paradox in heart failure. Prog Cardiovasc Dis 2016;58:393-400.

3 Horwich TB, Fonarow GC, Clark AL. Obesity and the obesity paradox in heart failure. Prog Cardiovasc Dis 2018;61:151-6.

4 Shah R, Gayat E, Januzzi JL, et al. Body mass index and mortality in acutely decompensated heart failure across the world: a global obesity paradox. J Am Coll Cardiol 2014;63:778-85.

5 Vest AR, Wu Y, Hachamovitch R, et al. The heart failure Overweight/ Obesity survival paradox: the missing sex link. JACC Heart Fail 2015;3:917-26.
6 Padwal R, McAlister FA, McMurray JJV, et al. The obesity paradox in heart failure patients with preserved versus reduced ejection fraction: a meta-analysis of individual patient data. Int $J$ Obes 2014;38:1110-4.

7 Stokke TM, Hasselberg NE, Smedsrud MK, et al. Geometry as a Confounder when assessing ventricular systolic function: comparison between ejection fraction and strain. J Am Coll Cardiol 2017;70:942-54.

8 Lavie CJ, Milani RV, Ventura HO, et al. Disparate effects of left ventricular geometry and obesity on mortality in patients with preserved left ventricular ejection fraction. Am J Cardiol 2007;100:1460-4.

9 Patel DA, Lavie CJ, Artham SM, et al. Effects of left ventricular geometry and obesity on mortality in women with normal ejection fraction. Am J Cardiol 2014;113:877-80.

10 Lavie CJ, Ventura HO. The obesity paradox in heart failure: is it all about fitness, fat, or sex? JACC Heart Fail 2015;3:927-30.

11 Park JJ, Park J-B, Park J-H, et al. Global longitudinal strain to predict mortality in patients with Acute Heart Failure. J Am Coll Cardiol 2018;71:1947-57.

12 Carnethon MR, De Chavez PJD, Biggs ML, et al. Association of weight status with mortality in adults with incident diabetes. JAMA 2012;308:581-90.

13 Willett WC, Hu FB, Thun M. Overweight, obesity, and all-cause mortality. JAMA 2013;309:1681.

14 Choo V. WHO reassesses appropriate body-mass index for Asian populations. Lancet 2002;360:235.

15 Lang RM, Badano LP, Mor-Avi V, et al. Recommendations for cardiac chamber quantification by echocardiography in adults: an update from the American Society of echocardiography and the European association of cardiovascular imaging. Eur Heart $J$ Cardiovasc Imaging 2015;16:233-71.

16 Lee SE, Lee H-Y, Cho H-J, et al. Clinical characteristics and outcome of acute heart failure in Korea: results from the Korean acute heart failure registry (KorAHF). Korean Circ J 2017;47:341-53.

17 Wong CY, O'Moore-Sullivan T, Leano R, et al. Alterations of left ventricular myocardial characteristics associated with obesity. Circulation 2004;110:3081-7.

18 Lavie CJ, Milani RV, Ventura HO. Obesity and cardiovascular disease: risk factor, paradox, and impact of weight loss. J Am Coll Cardiol 2009;53:1925-32.

19 Obokata M, Reddy YNV, Pislaru SV, et al. Evidence supporting the existence of a distinct obese phenotype of heart failure with preserved ejection fraction. Circulation 2017;136:6-19.

20 WHO Expert Consultation. Appropriate body-mass index for Asian populations and its implications for policy and intervention strategies. Lancet 2004;363:157-63.

21 Garcia-Moll X. Obesity and prognosis: time to forget about metabolically healthy obesity. Eur Heart $J$ 2018;39:407-9.

22 Mørkedal B, Vatten LJ, Romundstad PR, et al. Risk of myocardial infarction and heart failure among metabolically healthy but obese individuals: Hunt (Nord-Trøndelag health study), Norway. J Am Coll Cardiol 2014;63:1071-8.

23 Turkbey EB, McClelland RL, Kronmal RA, et al. The impact of obesity on the left ventricle: the multi-ethnic study of atherosclerosis (MESA). JACC Cardiovasc Imaging 2010;3:266-74.

24 Lavie CJ, Milani RV, Ventura HO. Adipose Composition and Heart Failure Prognosis: Paradox or Not? J Am Coll Cardiol 2017;70:2750-1.

25 Tsujimoto T, Kajio H. Abdominal Obesity Is Associated With an Increased Risk of All-Cause Mortality in Patients With HFpEF. J Am Coll Cardiol 2017;70:2739-49.

26 Kennedy AB, Lavie CJ, Blair SN. Fitness or fatness: which is more important? JAMA 2018;319:231-2.

27 Lavie CJ, Ortega FB, Kokkinos P. Impact of physical activity and fitness in metabolically healthy obesity. J Am Coll Cardiol 2018;71:812-3.

28 McAuley PA, Keteyian SJ, Brawner CA, et al. Exercise capacity and the obesity paradox in heart failure: the fit (Henry Ford exercise testing) project. Mayo Clin Proc 2018;93:701-8.

29 Moholdt T, Lavie CJ, Nauman J. Sustained Physical Activity, Not Weight Loss, Associated With Improved Survival in Coronary Heart Disease. J Am Coll Cardiol 2018;71:1094-101.

30 Lavie CJ, Laddu D, Arena R, et al. Healthy weight and obesity prevention: JACC health promotion series. J Am Coll Cardiol 2018;72:1506-31.

31 Fletcher GF, Landolfo C, Niebauer J, et al. Promoting physical activity and exercise: JACC health promotion series. J Am Coll Cardiol 2018;72:1622-39. 